\title{
Correlation of EEG Slowing with Cognitive Domains in Nondemented Patients with Parkinson's Disease
}

\author{
Ronan Zimmermann ${ }^{a}$ Ute Gschwandtner ${ }^{a}$ Florian Hatz ${ }^{a}$ \\ Christian Schindler $^{b} \quad$ Habib Bousleiman $^{a}{ }^{b}$ Shaheen Ahmed ${ }^{a}$ \\ Martin Hardmeier ${ }^{a} \quad$ Antonia Meyer ${ }^{a}$ Pasquale Calabrese ${ }^{c}$ Peter Fuhr $^{a}$ \\ ${ }^{a}$ Department of Neurology, University Hospital Basel, b Swiss Tropical and Public Health \\ Institute, and 'Department of Molecular and Cognitive Neuroscience, University of Basel, \\ Basel, Switzerland
}

\section{Key Words}

Mild cognitive impairment - Memory · Executive functions · Mental processes ·

Neuropsychology

\begin{abstract}
Background: Cognitive deficits in Parkinson's disease (PD) are heterogeneous and can be classified into cognitive domains. Quantitative EEG is related to and predictive of cognitive status in PD. In this cross-sectional study, the relationship of cognitive domains and EEG slowing in PD patients without dementia is investigated. Methods: A total of 48 patients with idiopathic PD were neuropsychologically tested. Cognitive domain scores were calculated combining Z-scores of test variables. Slowing of EEG was measured with median EEG frequency. Linear regression was used for correlational analyses and to control for confounding factors. Results: EEG median frequency was significantly correlated to cognitive performance in most domains (episodic long-term memory, rho $=0.54$; overall cognitive score, rho $=0.47$; fluency, rho $=0.39$; attention, rho $=0.37$; executive function, rho $=0.34$ ), but not to visuospatial functions and working memory. Conclusion: Global EEG slowing is a marker for overall cognitive impairment in PD and correlates with impairment in the domains attention, executive function, verbal fluency, and episodic long-term memory, but not with working memory and visuospatial functions. These disparate effects warrant further investigations.
\end{abstract}


Zimmermann et al.: Correlation of EEG Slowing with Cognitive Domains in Nondemented Patients with Parkinson's Disease

\section{Introduction}

Cognitive impairment in Parkinson's disease (PD) is heterogeneous [1, 2]. The identification of subtypes of cognitive impairment in PD is probably relevant for disease course and clinical management $[3,4]$. The Movement Disorders Society (MDS) task force proposes the classification of neuropsychological tests into different cognitive domains, namely attention, working memory, executive functions, language, memory, and visuospatial functions. This classification needs validation.

A biomarker-based investigation of cognitive subtypes might lead to a better understanding and the definition of clinically useful cognitive entities [5]. Slowing of oscillatory brain activity (EEG and MEG) has been proposed as a surrogate marker of cognitive dysfunction [6-11], and has also been identified as a predictive marker of dementia in PD [12, 13]. However, at present, it is not clear whether the EEG slowing in PD is a marker of overall cognition, or whether it is related to specific cognitive domains. It is important to appraise the contribution of EEG slowing to neuropsychological diagnostic and prognosis. The present study investigates the relationship between EEG slowing and domain-specific cognitive performance. It is hypothesized that the EEG slowing might be related to specific cognitive domains. In the interest of a straightforward hypothesis and out of many possible parameters, only EEG median frequency, which is a robust and promising EEG measure, is investigated in this study.

\section{Materials and Methods}

\section{Participants}

From May 2011 to January 2013, 53 patients with PD were recruited from the outpatient clinic for movement disorders of the University Hospital Basel or through advertisements in the magazine of the Swiss Parkinson's Disease Association. Patients were included if they fulfilled the UK Parkinson's Disease Brain Bank criteria [14]. Patients were excluded if they had an MMSE score of $<24$, other severe neurological conditions, or insufficient knowledge of the German language. Five patients were excluded due to EEG quality (see below), leaving 48 participants for statistical analysis. Patient characteristics were as follows: mean age $67.6 \pm 8.2$ years, 16 females and 32 males, $14.6 \pm 3$ years of education (school and main formation after school), $8.4 \pm 4$ years of disease duration (starting with first symptoms), levodopa-equivalent dose (LED) $688 \pm 467$, and Unified Parkinson's Disease Rating Scale part III (UPDRS III) score $14.8 \pm 11.4$ on medication.

Standard Protocol Approvals, Registrations, and Patient Consents

The study was approved by the local ethics committee (Ethikkommission beider Basel, ref. No.: 135/11). All participants were fully informed of the nature of the study and gave their written informed consent.

\section{Neuropsychological Assessments}

The patients were tested with a neuropsychological battery, distributed over two sessions because of the copious tests applied. The tests were administered in a fixed order. Data which could not be collected during these sessions were subsequently collected during the next 5-6 weeks, thus avoiding missing data. The tests were assigned to different cognitive domains based on their content (attention, working memory, executive functions, verbal fluency, episodic memory, and visuospatial functions). Raw scores were Z-transformed and, if necessary, inverted in order to achieve an overall positive metric (i.e. greater values representing a better performance). Z-scores of test variables (derived from raw scores without correction for demographic variables) were averaged per each domain to obtain domain scores. An overall test score was calculated by averaging the Z-score of all test variables, regardless of the domain ('overall cognitive score'). The tests and their domain attribution are summarized below, and are described in Strauss et al. [15], if not indicated otherwise. 
Zimmermann et al.: Correlation of EEG Slowing with Cognitive Domains in Nondemented Patients with Parkinson's Disease

Attention and Information Processing Speed

- Test of Attentional Performance (TAP) [16] - Alertness: (1) reaction time with alerting sound / (2) reaction time without alerting sound

- $\quad$ TAP - Divided Attention: (3) reaction time to auditive stimuli / (4) reaction time to visual stimuli / (5) omissions

- Stroop Color-Word Test: (6) time for color naming

- $\quad$ Trail-Making Test: (7) time for part A

Executive Functions

- Stroop Color-Word Test: (1) time for interference task divided by time for color naming

- Trail-Making Test: (2) time for part B divided by time for part A

- Wisconsin Card Sorting Test: (3) correct categories / (4) number of errors

Fluency

- Semantic verbal fluency test: (1) correct answers

- Phonemic verbal fluency: (2) correct answers

- $\quad$ Five-Point Test: (3) correct answers

Verbal and Figural Episodic Memory

- $\quad$ Rey-Osterrieth Complex Figure: (1) savings (immediate recall divided by copy)

- California Verbal Learning Test: (2) trial 1 / (3) trial 5 / (4) savings / (5) discriminability

Working Memory

- TAP - Working Memory (2-back task): (1) omissions

- Corsi blocks from the German version of the Revised Wechsler Memory Scale: (2) correct forwards / (3) correct backwards

- Digit span from the German version of the Revised Wechsler Memory Scale: (4) correct forwards / (5) correct backwards

Visuospatial Functions

- (1) Block Design Test

- (2) Rey-Osterrieth Complex Figure Copy

The patients' performance compared to age-, gender-, and education-corrected normative data of 604 healthy subjects [17] is shown in online supplementary figure e-1 (for all online suppl. material, see www. karger.com/doi/10.1159/000370110). On average, the patients had only mild deficits, i.e. the median performance was $<0.5$ standard deviations (below the performance of healthy control subjects in all domains).

EEG Data

A continuous eyes-closed, resting-state, 256-channel EEG of approximately $15 \mathrm{~min}$ was recorded (Netstation 300; EGI Inc., Eugene, Oreg., USA). The sampling rate was 1,000 Hz, and the reference electrode was $\mathrm{Cz}$ and re-referenced to average. Artifact-free segments of $>35 \mathrm{~s}$ were visually selected. EEGs were filtered (2,500 order least-square filter; band pass: $0.5-70 \mathrm{~Hz}$, notch: $50 \mathrm{~Hz})$, bad electrodes were automatically detected (using TAPEEG software [18]), and visually checked for plausibility. Artifacts such as ECG and eye blinks were detected and removed by application of an independent component analysis. Channels with bad activations were interpolated (spherical spline method). Frequency analysis was performed ('Welch' method [19]; sliding window of $4 \mathrm{~s}$ with $80 \%$ Hanning window and detection of bad windows with automated routines [18]). Median frequency was obtained from occipital electrodes. Out of 53 patients, 1 patient was excluded because of insufficient artifact-free material. Additionally, 4 patients were excluded based on the EEG quality criteria described in Hatz et al. [18].

EEG median frequency was used as primary outcome measure, since it is highly correlated with EEG peak frequency (background rhythmic frequency) ( $\mathrm{rho}=0.91 ; \mathrm{p}<0.001$; Spearman's rank correlation), and has a better test-retest reliability than the peak frequency [18].

Statistical Procedure: Correlation of EEG Slowing with Cognitive Domains

Statistical analyses were performed with R [20] and Stata [21]. The level of statistical significance was set at $\mathrm{p}=0.05$. 
Dementia

and Geriatric

Cognitive Disorders

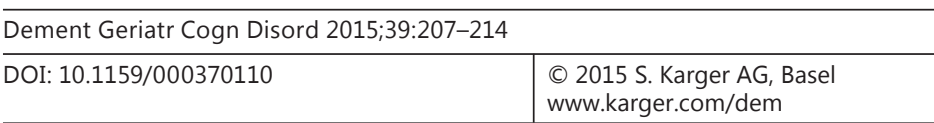

Zimmermann et al.: Correlation of EEG Slowing with Cognitive Domains in Nondemented Patients with Parkinson's Disease

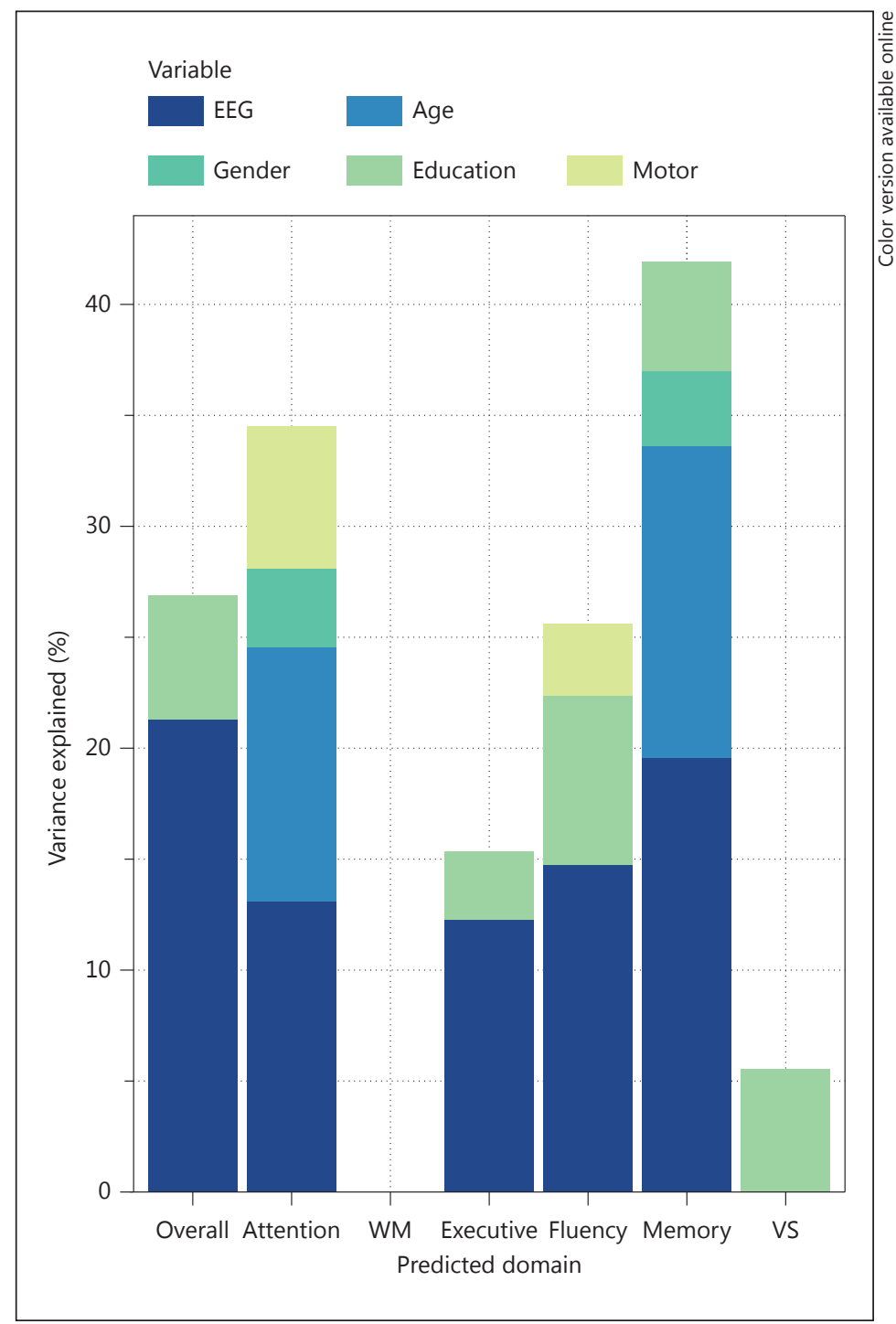

Fig. 1. Prediction of cognitive domains. Results of the linear regression analyses. The variance of the cognitive domain scores that is explained by EEG slowing (EEG) and the confounding variables is shown. The slower the EEG, the worse the performance on all neuropsychological variables. For working memory (WM) and visuospatial functions (VS), EEG slowing was not a relevant predictor. Motor $=$ Motor symptoms measured by UPDRS III.

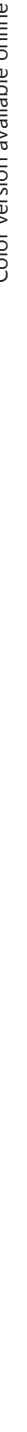

The 'overall cognitive score' was correlated with (and predicted by) EEG slowing, using a linear regression model. The potentially confounding factors age, gender, years of education, motor symptoms (UPDRS III), disease duration, and LED were used as additional predictors to control for their influence. Stepwise backwards elimination was used to consecutively eliminate nonpredictive variables. The same procedure was exploratively applied for all domain scores. The relative importance of the predictors was calculated with the package relaimpo [22] in R and plotted in a bar diagram (fig. 1).

Additionally, Spearman's rank correlations were calculated between the single neuropsychological test parameters and median frequency.

Finally, we tested whether cognitive domains, showing significant associations with EEG slowing (domain group A), could be statistically distinguished from cognitive domains without such associations (domain group B), based on the regression coefficients of EEG slowing. This was done using analysis of covariance for the six-dimensional outcome vector formed by our domain scores and testing, with a suitable linear contrast, whether the mean of regression coefficients of EEG slowing in domain group A differed significantly from the corresponding mean in domain group B. The homogeneity of regression coefficients of EEG slowing within the domain groups was assessed using the respective post hoc tests. 
Zimmermann et al.: Correlation of EEG Slowing with Cognitive Domains in

Nondemented Patients with Parkinson's Disease

Table 1. Correlation of median EEG frequency with cognitive domains and overall cognition

\begin{tabular}{|c|c|c|c|c|c|c|}
\hline Domain & EEG slowing & Age & Male gender & Education & Motor symptoms & Overall model \\
\hline Overall & $0.21(0.03-0.38)^{*}$ & & & & & $\mathrm{R}^{2}=0.11, \mathrm{p}=0.02$ \\
\hline Attention & $0.29(0.05-0.53)^{*}$ & $-0.04(-0.04$ to 0.63$)$ & $0.3(-0.04$ to 0.64$)$ & & $-0.01(-0.03 \text { to }-0.47)^{*}$ & $\mathrm{R}^{2}=0.35, \mathrm{p}<0.01$ \\
\hline Working memory & & & & & & NA \\
\hline Executive functions & $0.33(0.08-0.58)^{*}$ & & & $0.05(-0.02$ to 0.11$)$ & & $\mathrm{R}^{2}=0.15, \mathrm{p}=0.02$ \\
\hline Fluency & $0.45(0.17-0.74)^{*}$ & & & $0.1(0.02 \text { to } 0.17)^{*}$ & $-0.02(-0.04$ to 0.00$)$ & $\mathrm{R}^{2}=0.26, \mathrm{p}<0.01$ \\
\hline Memory & $0.31(0.08-0.53)^{*}$ & $-0.02(-0.04 \text { to } 0.00)^{*}$ & $-0.63(-0.65$ to 0.07$)$ & $0.06(0.01 \text { to } 0.11)^{*}$ & & $\mathrm{R}^{2}=0.42, \mathrm{p}<0.01$ \\
\hline Visuo-construction & & & & $0.06(-0.02$ to 0.15$)$ & & $\mathrm{R}^{2}=0.04, \mathrm{p}=0.13$ \\
\hline
\end{tabular}

The results of the regression analyses predicting cognitive domains and overall cognitive score by EEG median frequency and potentially confounding factors are presented. Each row represents a linear regression model analysis predicting the variable in the first column. Columns $2-6$ show estimates of the predictors with confidence intervals in parentheses. The last column shows the overall model parameters $\mathrm{R}^{2}$ and the $\mathrm{p}$ values. NA $=$ Not available. ${ }^{*}$ Significant estimate, $\mathrm{p}<$ 0.05 .

\section{Results}

Correlation of Cognitive Domains and Median EEG Frequency

For statistical values of the linear regression analyses, see table 1 . The overall cognitive score correlated positively with EEG median frequency. Age and education were relevant confounding factors.

The domain scores for attention, executive functions, fluency, and episodic long-term memory were all positively correlated with the EEG median frequency. In contrast, working memory and visuospatial functions did not correlate with the EEG median frequency. The overall cognitive score and episodic long-term memory showed the strongest correlation with this parameter (see online suppl. fig. e-2 for more details). Although age, gender, education, and motor symptoms had an effect on the cognitive domains, the correlation of cognitive domains and EEG slowing was independent from these confounders. Figure 1 displays the different cognitive domain predictors in their relative importance, and figure 2 shows Spearman's rank correlations of EEG parameters with single test parameters.

The mean of significant beta estimates (attention, executive functions, fluency, and episodic long-term memory) differed significantly from the mean of nonsignificant estimates (visuospatial abilities and working memory; $\mathrm{p}=0.02$ ). Furthermore, there was no indication of heterogeneity among significant or nonsignificant beta estimates ( $p=0.46$ and 0.99 , respectively).

\section{Discussion}

The aim of the study was to investigate whether EEG slowing (measured by EEG median frequency) is related to overall cognitive performance or to specific cognitive domains in nondemented patients with PD. The EEG median frequency correlates positively with attention, executive functions, fluency, and episodic long-term memory performance. The EEG median frequency has the strongest predictive power for overall cognition.

Taken together, our results are in line with other studies reporting EEG slowing as a surrogate marker for cognitive decline in patients with PD [6-11]. The lack of correlation between EEG power with working memory and visuospatial functions suggests that these domains are regulated at least in part by other mechanisms than are attention, episodic longterm memory, fluency, and executive functions. Thus, although EEG slowing is related to a number of cognitive domains and can therefore be seen as an overall cognitive parameter in PD, it also shows some specificity. 
Zimmermann et al.: Correlation of EEG Slowing with Cognitive Domains in

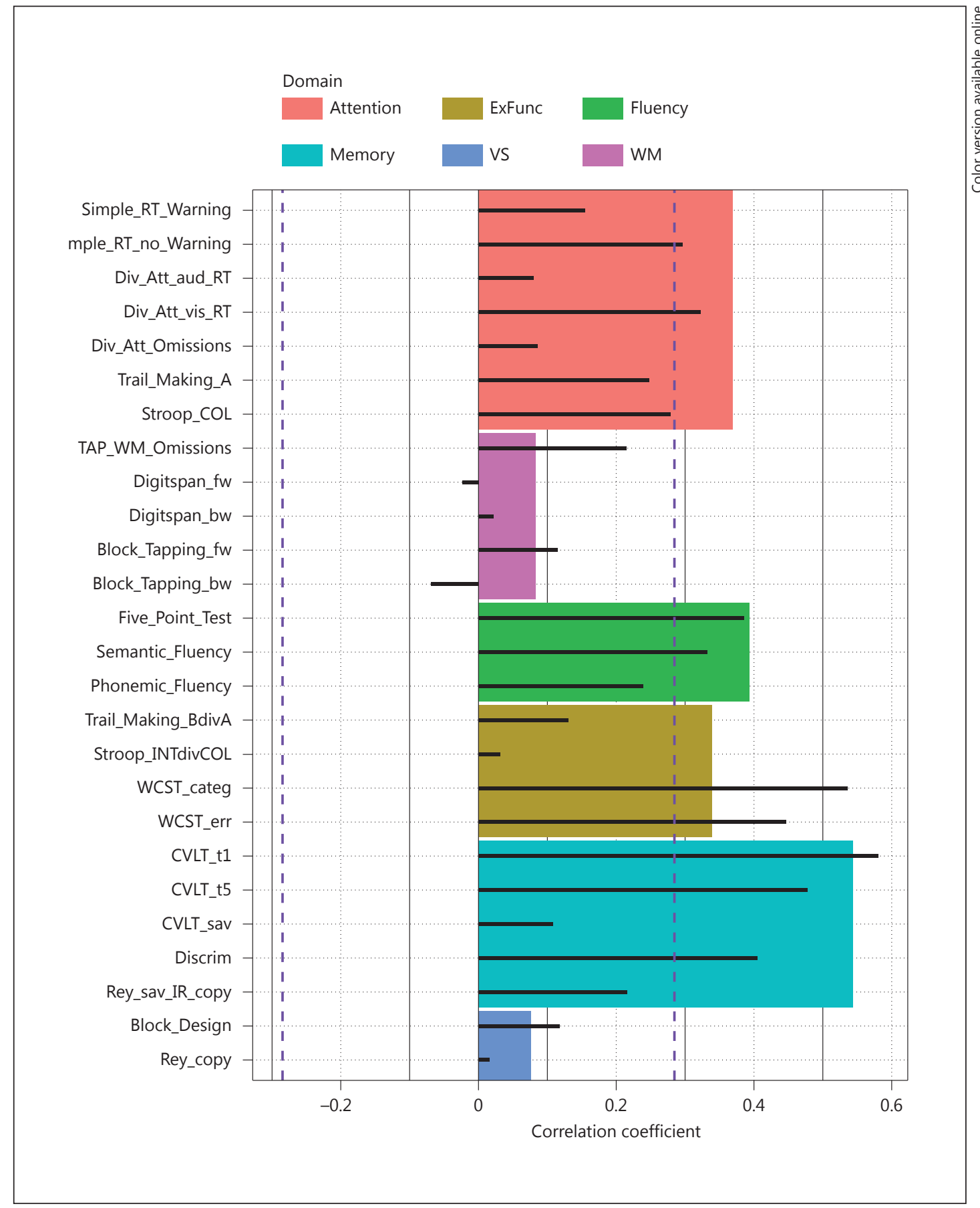

Fig. 2. Correlation of EEG median frequency with single test variables and cognitive domains. The bars represent Spearman's rank correlation coefficients of neuropsychological tests with EEG slowing (median frequency). For full names of neuropsychological tests, see the section Neuropsychological Assessments. Colored bars represent correlations of EEG slowing with domain scores. Single test bars are represented within their corresponding domain bar. Dashed lines represent the limits above which correlation coefficients are significant (without adjustment for multiple testing). RT = Reaction time. For colors, see the online version. 
Zimmermann et al.: Correlation of EEG Slowing with Cognitive Domains in

Nondemented Patients with Parkinson's Disease

The MDS task force proposed a domain-specific classification of cognitive abilities [4] according to clinically relevant phenotypes of cognitive impairment in PD. However, a domainspecific categorization of the neuropsychometric tool remains somewhat arbitrary. There is an ongoing debate about formal definitions and tests, which might best represent certain cognitive domains, and also separate them reliably. We tried to reproduce the domains proposed by the MDS task force, but treated attention and working memory as distinct domains. Our data might suggest that attention and working memory are rather separate domains, as working memory did not correlate with EEG slowing while attention did.

The patients in our study had a disease duration of 8.5 years and were, from this point of view, not in an early disease stage. In comparison to normative data of healthy subjects, however, the subjects in this study were cognitively only mildly affected. More specific results might evolve, as cognitive decline gets more pronounced. A selection bias towards rather cognitively intact patients is possible in this study, as patients were to some extent recruited by an advertisement and participated voluntarily. Furthermore, patients with an MMSE score of $<24$ were excluded to assure that the patients were able to participate in the extensive neuropsychological testing and to avoid stronger reciprocal influence of the cognitive domains. This approach also has the advantage of reducing the influence of possible sedative medication.

All patients were medicated and the influence of this medication on the EEG and the neuropsychological tests is unclear. However, LED did not show a relevant effect in our analyses. Different conceptions of cognitive domains are in use in the scientific literature, and the use of variable test sets might lead to variable results [1]. The present study did not investigate whether the association between EEG slowing and cognitive performance is similar in healthy subjects or specific to PD.

In conclusion, the substantial negative correlations of EEG slowing with overall cognition, as well as with episodic long-term memory, attention, fluency, and executive functions suggests that the EEG frequency analysis is potentially useful as an overall surrogate marker of performance in these domains in PD. Since the evolution and dynamics of cognitive decline in PD are still unresolved issues, biomarker-based investigations of cognitive domains in nondemented PD patients are important for a potential early detection of different forms of dementia and their clinical management.

\section{Acknowledgements}

We would like to thank the participating patients and their caregivers, Parkinson Schweiz, the Gossweiler Foundation, the Freiwillige Akademische Gesellschaft Basel, the Bangerter-Rhyner Foundation, the Swiss National Science Foundation, UCB Pharma AG, and Abbvie Pharma AG for supporting this study.

\section{References}

$>1$ Caviness JN, Driver-Dunckley E, Connor DJ, Sabbagh MN, Hentz JG, Noble B, et al: Defining mild cognitive impairment in Parkinson's disease. Mov Disord 2007;22:1272-1277.

$\checkmark 2$ Kehagia AA, Barker RA, Robbins TW: Neuropsychological and clinical heterogeneity of cognitive impairment and dementia in patients with Parkinson's disease. Lancet Neurol 2010;9:1200-1213.

-3 Litvan I, Aarsland D, Adler CH, Goldman JG, Kulisevsky J, Mollenhauer B, et al: MDS task force on mild cognitive impairment in Parkinson's disease: critical review of PD-MCI. Mov Disord 2011;26:1814-1824.

-4 Litvan I, Goldman JG, Tröster AI, Schmand BA, Weintraub D, Petersen RC, et al: Diagnostic criteria for mild cognitive impairment in Parkinson's disease: Movement Disorder Society Task Force guidelines. Mov Disord 2012;27:349-356.

5 Cholerton BA, Zabetian CP, Wan JY, Montine TJ, Quinn JF, Mata IF, et al: Evaluation of mild cognitive impairment subtypes in Parkinson's disease. Mov Disord 2014;29:756-764. 
Zimmermann et al.: Correlation of EEG Slowing with Cognitive Domains in Nondemented Patients with Parkinson's Disease

-6 Soikkeli R, Partanen J, Soininen H, Pääkkönen A, Riekkinen P: Slowing of EEG in Parkinson's disease. Electroencephalogr Clin Neurophysiol 1991;79:159-165.

7 Sinanović O, Kapidzić A, Kovacević L, Hudić J, Smajlović D: EEG frequency and cognitive dysfunction in patients with Parkinson's disease. Med Arh 2005;59:286-287.

-8 Caviness JN, Hentz JG, Evidente VG, Driver-Dunckley E, Samanta J, Mahant P, et al: Both early and late cognitive dysfunction affects the electroencephalogram in Parkinson's disease. Parkinsonism Relat Disord 2007;13: 348-354.

-9 Kamei S, Morita A, Serizawa K, Mizutani T, Hirayanagi K: Quantitative EEG analysis of executive dysfunction in Parkinson disease. J Clin Neurophysiol 2010;27:193-197.

10 Morita A, Kamei S, Mizutani T: Relationship between slowing of the EEG and cognitive impairment in Parkinson disease. J Clin Neurophysiol 2011;28:384-387.

11 Olde Dubbelink KTE, Stoffers D, Deijen JB, Twisk JWR, Stam CJ, Berendse HW: Cognitive decline in Parkinson's disease is associated with slowing of resting-state brain activity: a longitudinal study. Neurobiol Aging 2013; 34:408-418.

12 Klassen BT, Hentz JG, Shill HA, Driver-Dunckley E, Evidente VGH, Sabbagh MN, et al: Quantitative EEG as a predictive biomarker for Parkinson disease dementia. Neurology 2011;77:118-124.

$\checkmark 13$ Olde Dubbelink KTE, Hillebrand A, Twisk JWR, Deijen JB, Stoffers D, Schmand BA, et al: Predicting dementia in Parkinson disease by combining neurophysiologic and cognitive markers. Neurology 2014;82:263-270.

14 Gibb WR, Lees AJ: The relevance of the Lewy body to the pathogenesis of idiopathic Parkinson's disease. J Neurol Neurosurg Psychiatry 1988;51:745-752.

15 Strauss E, Sherman EMS, Spreen O: A Compendium of Neuropsychological Tests : Administration, Norms, and Commentary, ed 3. Oxford, Oxford University Press, 2006.

16 Zimmermann P, Fimm B: Testbatterie zur Aufmerksamkeitsprüfung. Herzogenrath, Psytest Psychologische Testsysteme, 2007.

$\checkmark 17$ Berres M, Zehnder A, Bläsi S, Monsch AU: Evaluation of diagnostic scores with adjustment for covariates. Stat Med 2008;27:1777-1790.

18 Hatz F, Hardmeier M, Bousleiman H, Rüegg S, Schindler C, Fuhr P: Reliability of fully automated versus visually controlled pre- and post-processing of resting-state EEG. Clin Neurophysiol 2014, Epub ahead of print.

19 Welch P: The use of fast Fourier transform for the estimation of power spectra: a method based on time averaging over short, modified periodograms. IEEE Trans Audio Electroacoustics 1967;15:70-73.

20 R Core Team: R: A Language and Environment for Statistical Computing. Vienna, R Foundation for Statistical Computing, 2012. http://www.R-project.org/.

21 StataCorp: Stata Statistical Software: Release 12. College Station, StataCorp LP, 2011. http://www.stata.com/ (accessed November 12, 2014).

22 Grömping U: Relative importance for linear regression in R: the package relaimpo. J Stat Softw 2006;17:1-27. 